\title{
Automated, Real-Time Estimation of Pile Bearing Capacity Via Seismic Measurements
}

\author{
Chung-Ham Yang H. Randolph Thomas
}

\author{
Department of Civil Engineering \\ Pennsylvania State University \\ University Park, PA 16802
}

\begin{abstract}
This research is aimed at developing a seismic measurement based method for estimating pile bearing capacity. By modeling the rigid body motion of the pilesoil system, the pile bearing capacity is analytically derived as a function of mass, natural frequency, damping ratio, and soil quake. This paper presents the procedure of extracting the natural frequency and the damping ratio from ground motion trace (also known as seismic waveform).

The model was examined using field data collected from a bridge foundation project. The estimated bearing capacities of the two test piles are close to the estimations by means of the well-known Case method. The results demonstrate the applicability of the model in estimating pile bearing capacity for the case project.
\end{abstract}

\section{Introduction}

The examination of bearing capacity is the major concern in the field control of pile construction. Two categories of methods, blow counts and stress wave analyses, prevail in today's practice.

Blow counts, which is the hammer blows required for each foot of pile penetration, has long been used as the examination criteria of bearing capacity [1]. The relation between bearing capacity and blow counts is predetermined and is generally represented in terms of a bearing graph as shown in Figure 1(a). With measured blow counts, the bearing capacity can then be determined. The blow counts approach requires no instrumentation and does not interrupt the pile driving operation. Therefore, piles installed at a given site can be examined, and a sitewide examination can be implemented.

The use of blow counts for site-wide examination is based on the assumption that the soil conditions and the driving energy for each blow is the same, or at least similar throughout the site. In reality, soil conditions often vary at a site and the energy delivered by each hammer blow is not the same. The use of predetermined bearing graph might lead to a false estimation. This is illustrated in Figure $1(b)$ where a same number of hammer blows represents different bearing capacity at two locations. (a)

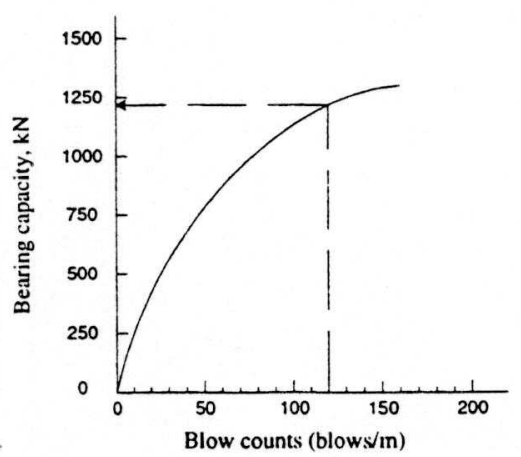

(b)

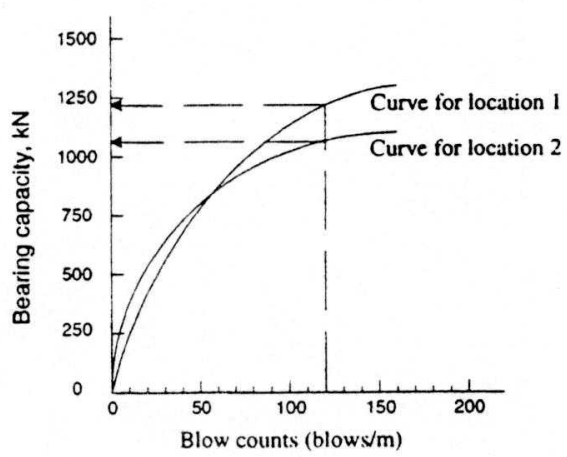

Fig. 1. (a) Bearing graph (b) Variation of bearing capacity at two locations 


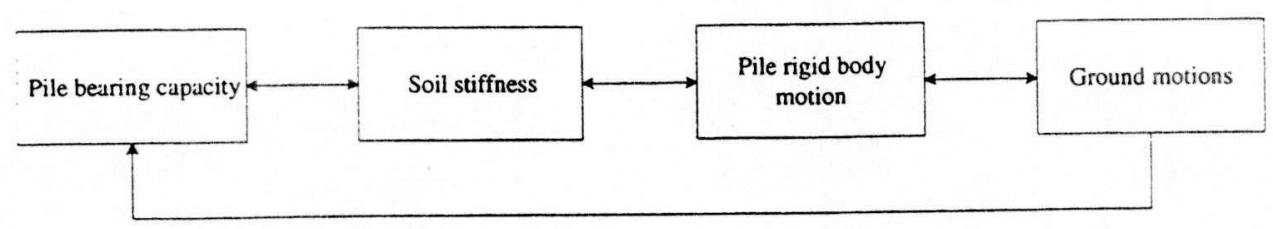

Fig. 2. Rationale of the seismic measurement based method

During the past few decades, stress wave theory has been applied to the estimation of pile bearing capacity [2]. The pile is considered as an elastic bar, and a stress wave is generated from the hammer impact on the pile head. The stress wave is transmitted in terms of pile particle motion. The stress wave theory states that the force and the velocity of the pile particle are proportional before stress wave reflections arrive at the pile top from resistance effects. Resistance effects cause the force to increase relative to the velocity. By characterizing the force and velocity measurements, the soil resistance can then be estimated.

The stress wave analyses are based on measurements made on the pile. Typically, the accelerometer and the strain gauge are instrumented to the pile shaft. This onpile instrumentation is expensive and it interrupts the pile driving operation. The high cost and interruptive nature of the stress wave based methods imposes practical limits on the number of piles to be tested, and the site-wide examination is not practically possible. This limited amount of tested piles may not reliably represent the rest of piles that are driven through a variety of soil conditions.

In summary, the blow counts approach can be applied to every piles at a site, but the results are subject to inaccuracy. Although the stress wave analyses produces much reliable results, the application to every piles is not practically possible.

\section{Rationale of the research}

A seismic measurement based method is conceived to remedy the shortcomings of current practices. As shown in Figure 2, the bearing capacity of the pile is a function of the stiffness of the soil stratum that bears the pile. The soil stiffness dictates the manner in which the pile moves. The pile motion interacts with surrounding soils, producing ground motions. It is hypothesized that the pile bearing capacity can be estimated by characterizing the ground motions.

The measurement of ground motions needs no on-pile instrumentation. and does not interrupt the driving operation. This enables every pile at a given site to be tested. The inaccuracy resulting from the variation of soil conditions can then be minimized.

\section{Scope of the research}

The scope of the research is to develop the theoretical basis of estimating pile bearing capacity via seismic measurements. The pile bearing capacity is expected to be analytically related to ground motion by modeling the pile rigid body motion. The analytical model will be tested using field data, and the applicability of the model will be discussed.

\section{Theoretical basis}

\subsection{Pile rigid body motion model}

The ground motions are disturbed by the pile motion which consists of both in-pile deformation and rigid body motion. The in-pile deformation is due to the elasticity of pile material, and the rigid body motion is the result of soil deformation. The soil deformation is complex in nature meaning that it involves both elastic and plastic process. Smith [3] suggests that the elastoplastic characteristics of soils can be idealized as shown in Figure 3. Upon the impact of the hammer, the soil deforms following a trace $\mathrm{OABC}$ where line $\mathrm{OA}$ is a straight line with a constant slope $k$, and the line $A B$ is parallel to the $x$ axis, and the line $B C$ is parallel to line OA (i.e., the slope of two lines is the same).

According to Figure 3 , the soil is expected to compress linearly to the quake point $A$, at which point the ultimate soil resistance, $R_{\mathrm{u}}$, is developed. The OA trace is designated as phase 1 . When the impact load exceeds the ultimate soil resistance, $R_{u}$, the soil fails plastically following the trace $\mathrm{AB}$. This is designated as phase 2 . During continued application of the load, the soil retains a resistance equal to $R_{u}$. Upon removal of the force, the soil 
oscillates vertically until the impact energy dissipates due to the soil damping. This is designated as phase 3. A full recovery of elastic deformation equal to $Q$ occurs, and the permanent plastic deformation, $S$, is retained.

The elastic nature of the soil can be represented by a linear spring [4.5]. The stiffness constant of the spring, which is the amount of force required to cause a unit of spring deformation, is the slope of the elastic deformation trace $\mathrm{OA}$ and $\mathrm{BC}$. The spring resistance increases until it reaches a displacement $Q$, known as the quake. At that point, the spring resistance reaches the ultimate resistance, $R_{u}$, which equals to the product of quake and soil stiffness. That is,

$$
R_{u}=k Q
$$

\subsection{Mathematical formulation}

As observed in Figure 3, the elastic motion occurs in phase 1 and 3 , therefore the spring constant $k$ can be determined from phase 1 and 3 . The elastic motion in phase 1 is a forced motion, and the force is not deterministic. No deterministic formulation for $\mathrm{k}$ can be derived. In phase 3 , the pile is in free oscillation. The spring constant can be derived as [6]

$$
k=\frac{4 \pi^{2} m f_{d}^{2}}{1-D^{2}}
$$

where $\mathrm{k}$ = soil spring constant, $\mathrm{N} / \mathrm{m}$

$\mathrm{m}=$ vibrating mass, $\mathrm{kg}$

$\mathrm{f}_{\mathrm{d}}=$ damped natural frequency, $\mathrm{Hz}$ or $1 / \mathrm{sec}$

$\mathrm{D}=$ damping ratio

Substituting Equation (2) into (1), the ultimate soil resistance ( $=$ bearing capacity) is as follows.

$$
R_{u}=k Q=\frac{4 \pi^{2} m f_{d}^{2}}{1-D^{2}} Q
$$

where $\mathrm{R}_{\mathrm{u}}=$ ultimate soil resistance = bearing capacity, $\mathrm{N}$

$\mathrm{k}=$ soil spring constant, $\mathrm{N} / \mathrm{m}$

$\mathrm{m}=$ vibrating mass, $\mathrm{kg}$

$\mathrm{f}_{\mathrm{d}}=$ damped natural frequency, $\mathrm{Hz}$ or $1 / \mathrm{sec}$

$\mathrm{D}=$ damping ratio

$\mathrm{Q}=$ soil quake, $\mathrm{m}$

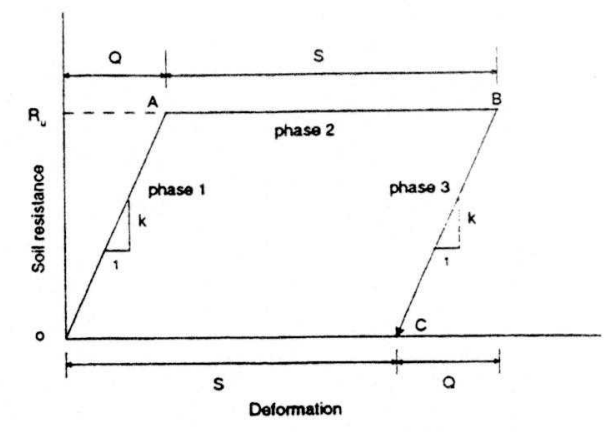

Fig. 3. Idealized load-deformation trace of soils

\section{Case study}

The proposed model was examined using data collected from a bridge foundation project. The pile for the project consisted of $7.2 \mathrm{~m}$ long HP $12 \times 53$ steel piles with a designed capacity of $1335 \mathrm{kN}$. The soil stratum consists of various layers of silty sand and silty clay overlying claystone bedrock. The piles were driven by a Vulcan 01 single acting air hammer.

\subsection{Data acquisition and signal processing}

The observation were made on two piles, TP1 and TP2, from the initial setting to driving refusal which takes place at penetration depth of $6 \mathrm{~m}$. The acceleration of vertical ground motion was measured by placing an accelerometer on a steel probe inserted into the ground. Preliminary studies indicate that the frequency of pile rigid body motion is in the range of 10 to $50 \mathrm{~Hz}$ [7]. In this research, a low-pass filter was used to remove signals with frequencies above $50 \mathrm{~Hz}$, and retain low frequency signals that correspond to rigid body motion. To be further processed in a digital instrument, the filtered signals were converted into digital format and displayed as a function of time.

\subsection{Seismic waveform}

The time domain display of the signals is termed seismic waveform. A typical seismic waveform which corresponds to a hammer blow is shown in Figure 4. Three distinct wave patterns are identified, and each corresponds to certain force origin. The force origins and the resulting waves are illustrated in Figure 5. Prior to ram-pile impact, the free fall of the ram shakes the guide frame. The vertical movement of the guide frame disturbs the ground, producing a series of ground waves. 


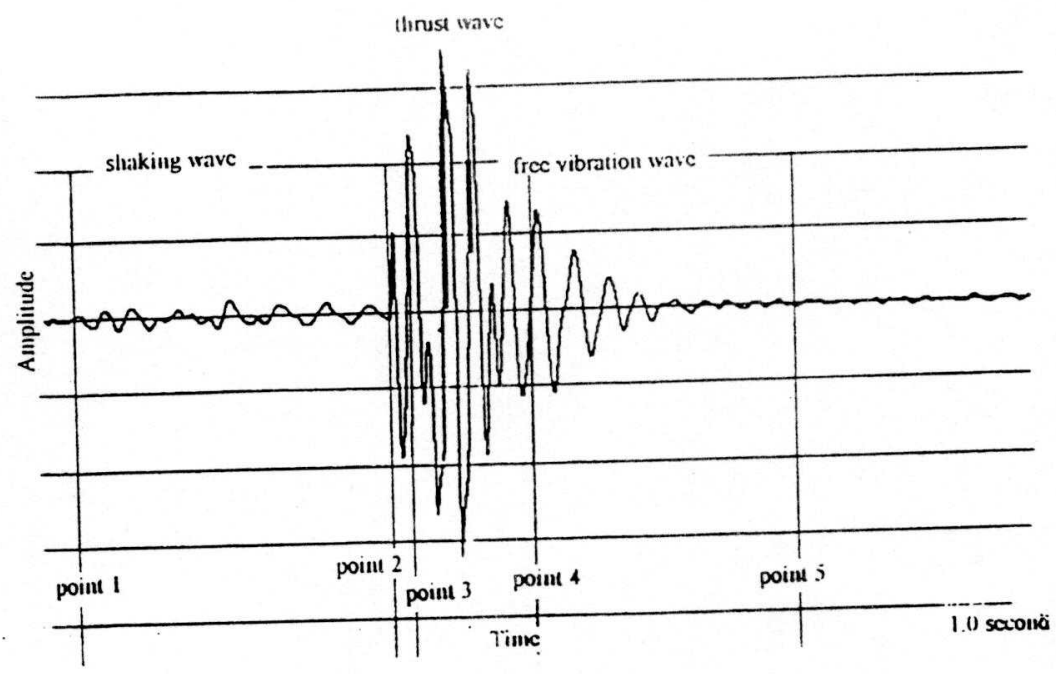

Fig. 4. Seismic waveform corresponding to a hammer blow

These waves are not significant in intensity and add up to a shaking wave. As shown in Figure 6(a), the shaking wave begins at point 1 and continues for certain time.

Upon impact of the ram, the pile thrusts downwards. The downward movement generates a series of ground waves. These waves are significant in intensity and add up to a thrust wave. As shown in Figure 6(b), the thrust wave begins at point 2 at which time the ram-pile impact takes place. It is noted that the early portion of the thrust wave overlaps with the latter portion of the shaking wave. The overlapped portion of the thrust wave is identified from point 2 to point 3 in both Figure 4 and $6(d)$.
Beginning at point 3 , the ram loses contact with the pile. There is no force applied to the pile after point 3 , and the pile freely oscillates. The free vibration of the pile disturbs the ground, producing a free vibration wave which possesses a pattern of damped sine wave. It is noted that the early portion of the free vibration wave overlaps with the latter portion of the thrust wave. The overlapped portion, which is from point 3 to point 4 in both Figure 4 and 6(d), displays no significant pattern of damped sinewave. This is because the thrust wave masks the free wave. After point 4, the thrust wave dies out, and the pattern of damped sinewave displays clearly.
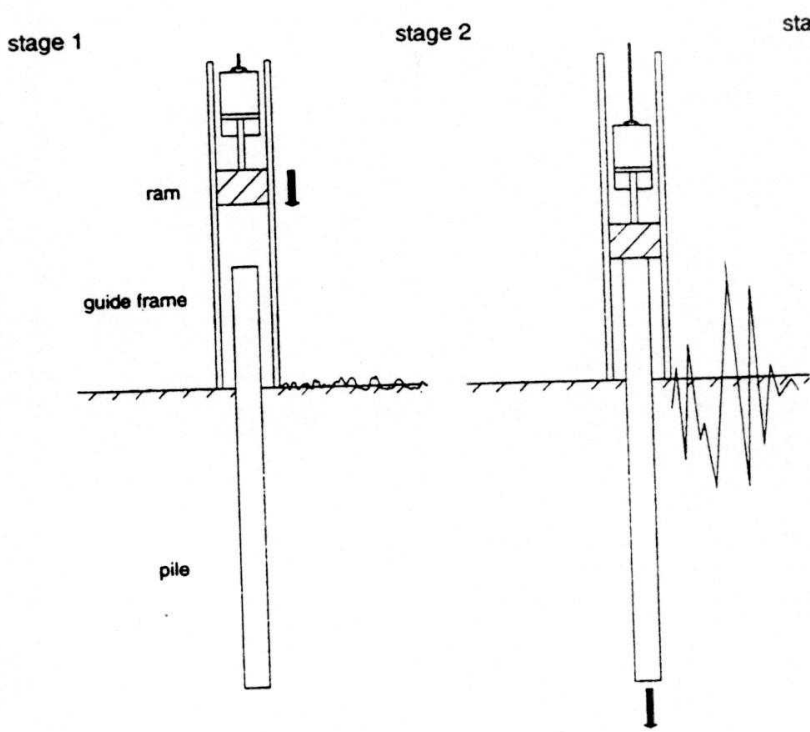

stage 3

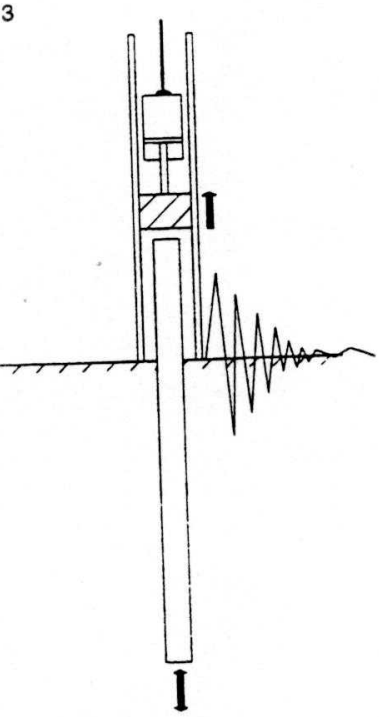

Fig. 5. Force origins and resulting ground waves 


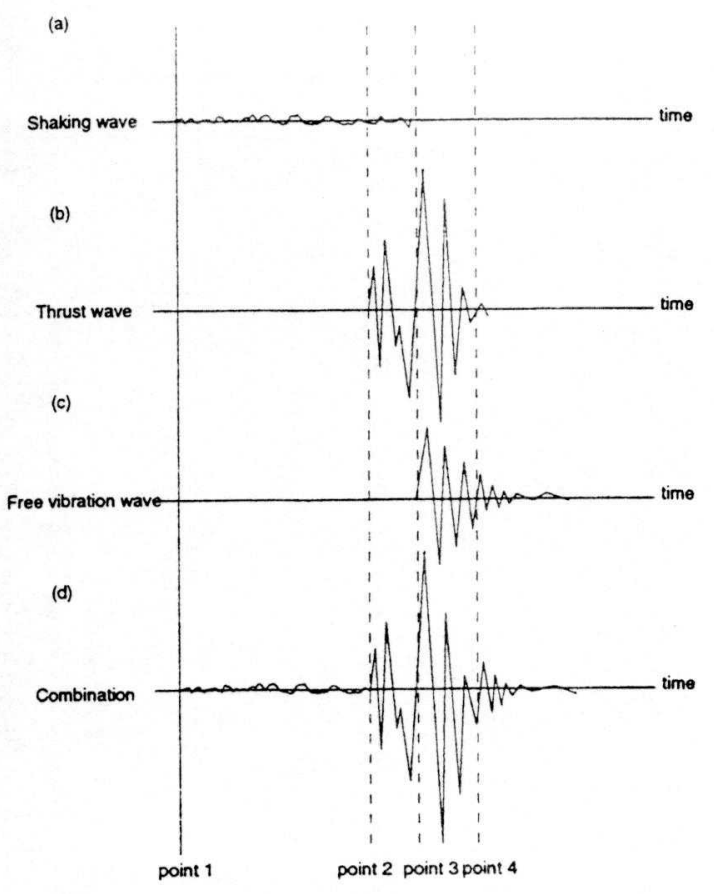

Fig. 6. (a) shaking wave (b) thrust wave (c) free vibration wave (d) combination

\subsection{Determination of natural frequency and damping ratio}

In Equation (3), the damped natural frequency and damping ratio are parameters that describe the free elastic motion of the pile. The seismic response to free elastic motion manifests itself as a free vibration wave from which the damped natural frequency and damping ratio can be determined. As shown in Figure 7, a logarithmic curve fits a sequence of peaks. The first peak that touches the curve is designated as $\mathrm{TC} 1$, and the second peak that touches the curve is designated as TC2. The damped natural frequency is the inverse of period which is the time period between $\mathrm{TC} 1$ and $\mathrm{TC} 2$ [5]. In this figure, the damped natural frequency is $27.82 \mathrm{~Hz}$. The damping ratio is a function of the ratio of amplitudes of two successive peaks [5]. In this figure, the damping ratio is calculated as 0.073 .

In summary, the procedure of determining damped natural frequency and damping ratio involves the identification of a free vibration wave, measurement of timing and amplitudes of peaks, and the calculation of parameters.

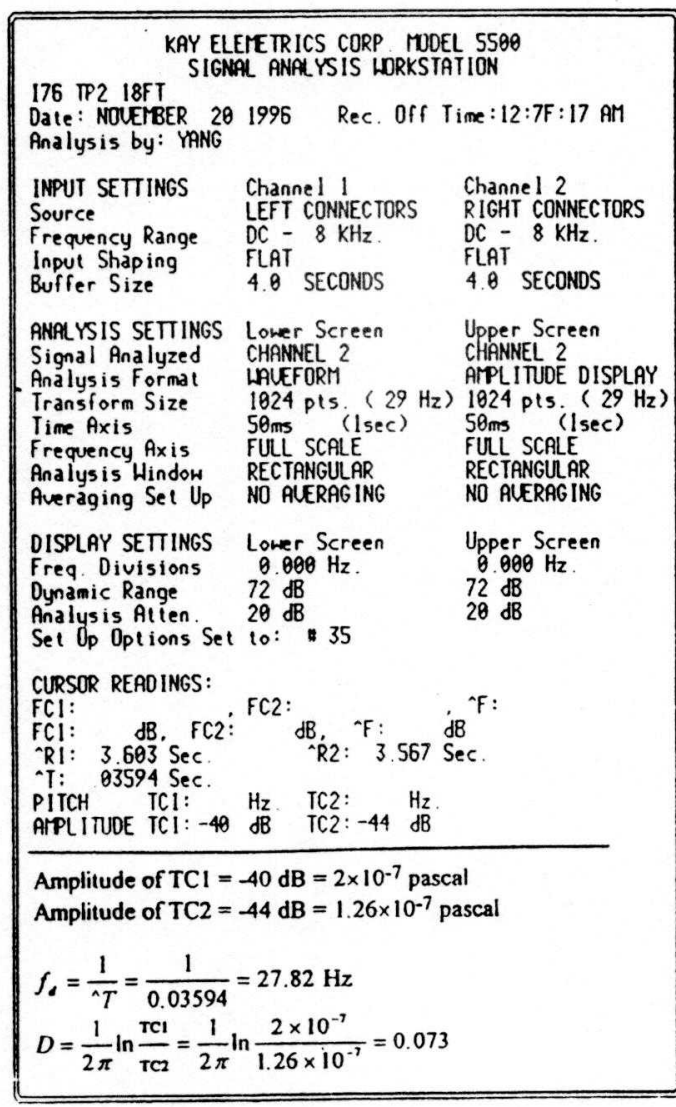

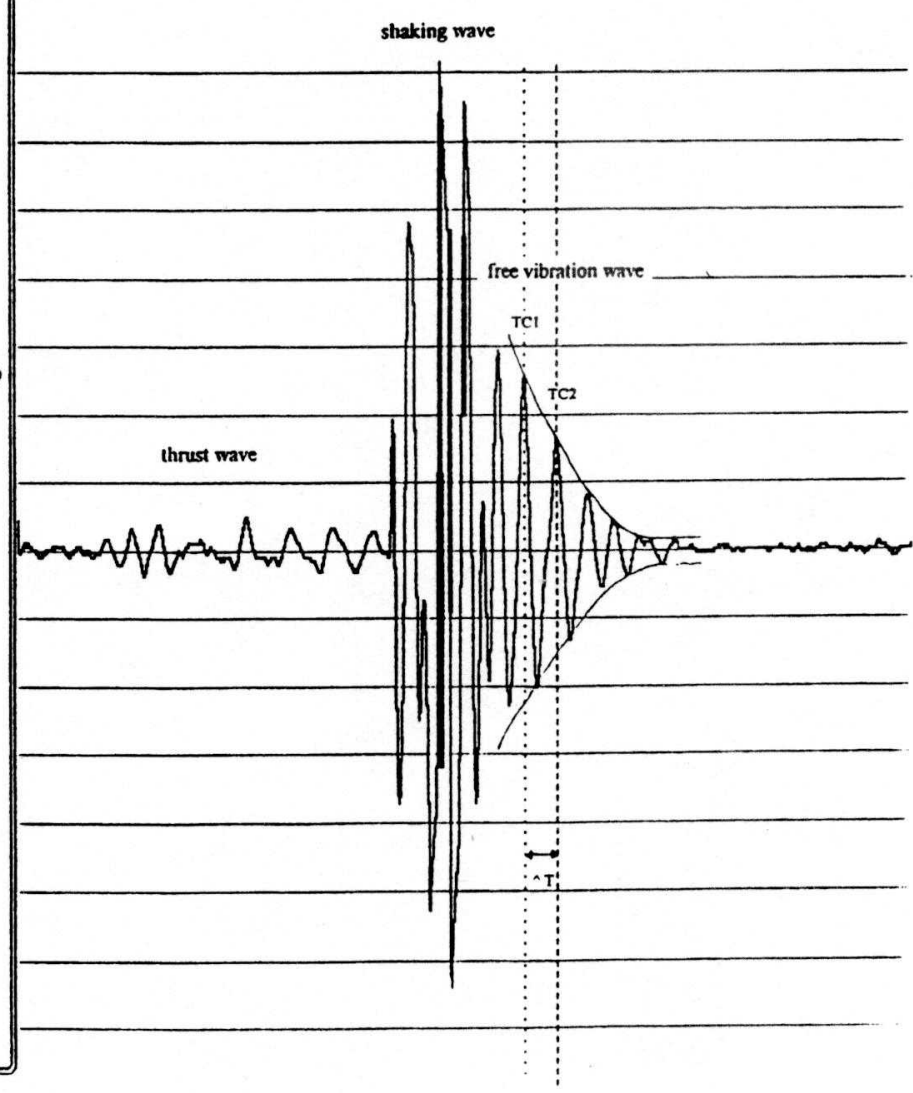

Fig. 7. Determination of damped natural frequency and damping ratio 
HP12 $\times 53$

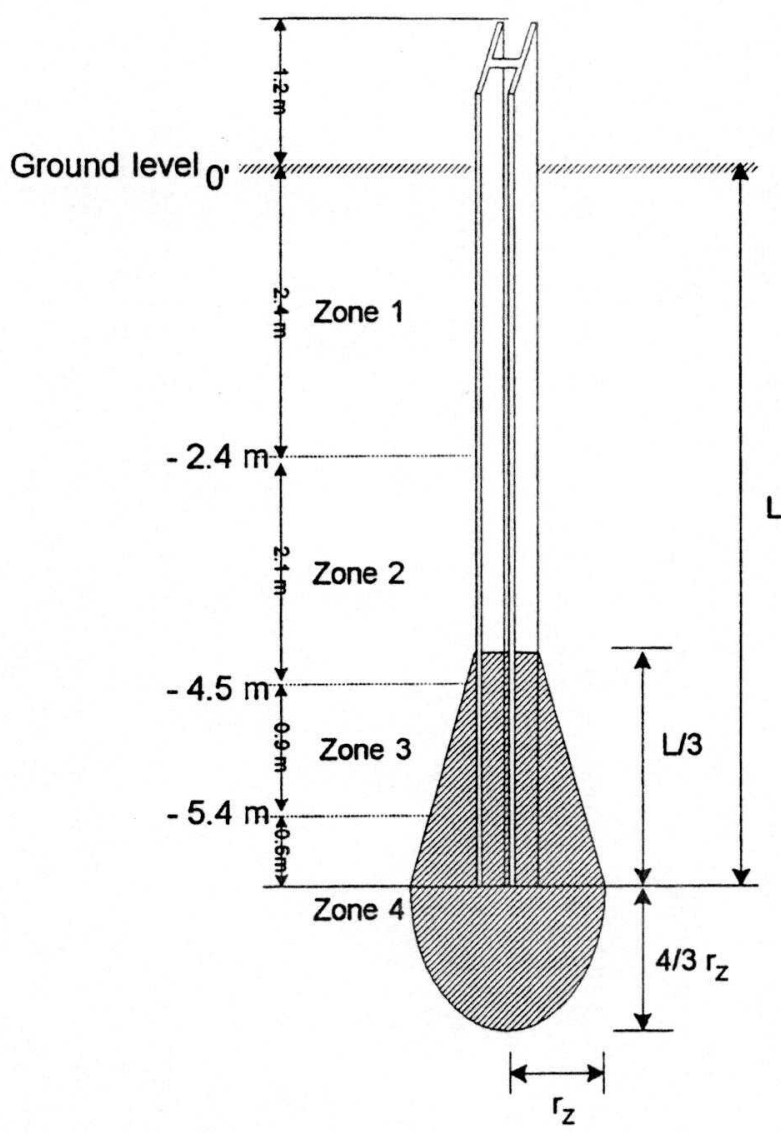

pile mass, $m_{1}$

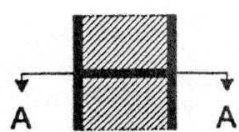

$$
\begin{aligned}
Z & =\left(\frac{L}{3} \times \tan 30^{\circ}\right)+\frac{d}{2} \\
& =\left(\frac{6}{3} \times \tan 30^{\circ}\right)+\frac{0.15}{2} \\
& =1.25 \mathrm{~m} \\
m_{1} & =\left(\frac{L}{3} \times W_{\text {pile }}+\frac{L}{3} \times A_{\text {soil }} \times \gamma_{\text {soil }}\right)+9.81 \\
& =\left(\frac{6}{3} \times 7745+\frac{6}{3} \times 0.082 \times 20421\right)+9.81=500 \mathrm{~kg} \\
m_{2} & =\frac{1}{3} \times\left[\frac{\pi}{9} r^{2}(L+16 \mathrm{Z})-\frac{L}{3} \frac{\pi d^{2}}{4}\right] \times \gamma_{\text {soil }}+981 \\
& =\frac{1}{3} \times\left[\frac{\pi}{9}(1.25)^{2}(6+16 \times 1.25)-\frac{6}{3} \frac{\pi(0.15)^{2}}{4}\right] \times 20421+981 \\
& =9820 \mathrm{~kg} \\
m & =m_{1}+m \\
& =500+9820 \\
& =10320 \mathrm{~kg}
\end{aligned}
$$

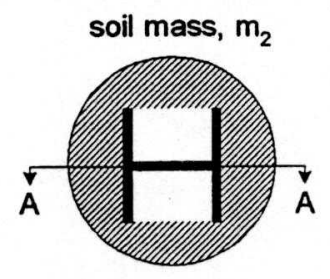

Fig. 8. Vibration mass at final depth of TP1 and TP2 


\subsection{Determination of vibrating mass and soil quake}

Besides the damped natural frequency and damping ratio, the vibrating mass and the soil quake need to be determined. The literature suggests a bulb shape formulation which takes both pile mass and soil mass into consideration [8]. The dimension of the bulb and the unit weights of soils are shown in Figure 8. At the penetration depth of $6 \mathrm{~m}$, the vibrating mass was calculated as 10320 $\mathrm{kg}$.

The determination of soil quake was not studied in this research. Typically, soil quake is estimated based upon soil properties or through wave equation analyses [2]. A wave equation based analysis, CAPWAP, was performed on the observed piles. The soil quake was determined as the value of $0.0038 \mathrm{~m}$, and this value was used as the estimate of the soil quake.

\subsection{Calculation of bearing capacity}

Using Equation (3), the bearing capacities of TP1 and TP2 at the final depth were calculated. The calculated values were compared with the results of the Case method, as summarized in Table 1. The percentages in the last column are the variations between the two estimations. The variation for TP1 is $13 \%$, and the variation for TP2 is $2 \%$. These small variations indicate that the results of the proposed procedure are close to the results obtained by the Case method.

\section{Table 1: Comparison of $\mathbf{R}_{\text {case }}$ and $\mathbf{R}_{\text {seismic }}$}

\begin{tabular}{|c|c|c|c|}
\hline Test Pile & $\mathrm{R}_{\text {case }}$ & $\mathrm{R}_{\text {seismic }}$ & Variation \\
& $(1)$ & $(2)$ & $\frac{(2)-(1)}{(1)} \times \%$ \\
\hline TP1 & $1556 \mathrm{kN}$ & $1767 \mathrm{kN}$ & +13 \\
\hline TP2 & $1356 \mathrm{kN}$ & $1390 \mathrm{kN}$ & +2 \\
\hline
\end{tabular}

\section{Implementation of automation}

The procedure of determining damped natural frequency and damping ratio can be encoded for the purpose of automation. The algorithms to be developed include the identification of a free vibration wave, measurement of timing and amplitudes of peaks, and the calculation of parameters and bearing capacity. More complex signal processing algorithms might be needed for signal enhancement and noise filtering. These algorithms can be easily encoded and executed in a conventional personal computer.

Other aspects of the implementation of automation include the automated signal measurement and transmission. It is expected that the ground motions are automatically measured and processed, and the bearing capacity can then be calculated in real-time. This automated procedure enables every pile at a site to be tested in a cost-effect and efficient manner. Also, the inaccuracy resulting from the variation of soil conditions can be minimized.

\section{Summary, conclusion, and future research}

This research developed the theoretical basis of estimating pile bearing capacity via seismic measurements. The pile-soil system is modeled as a mass-spring-sliderdashpot combination, and the pile bearing capacity is derived as a function of parameters that describe the elastic motion of the system. These parameters are mass, damped natural frequency, damping ratio and soil quake. The procedure of determining natural frequency and damping ratio from the seismic waveform was presented in this paper. It is expected that the procedure can be computerized.

The model was examined using field data collected from a bridge foundation project. The damped natural frequency and damping ratio were extracted from seismic waveforms, and the vibrating mass was estimated based on soil unit weight. The determination of the soil quake was not studied in this research. Instead, the soil quake that was estimated by means of CAPWAP analysis was used as the estimate of the soil quake. Given this value of soil quake, the proposed model produces bearing capacities close to the estimations obtained by the Case method.

In this research, the examination of the model was limited to piles at a site. Due to limited data, the generic applicability of the model in estimating pile bearing capacity can not be concluded. However, the significance of the results justifies intensive validation of the model to be made under various driving and soil conditions.

\section{References}

[1] D.P. Coduto, Foundation Design, Principles and Practices, Prentice Hall, 1994.

[2] G.G. Goble, F. Rausche, and G. E. Likins, "The Analysis of Pile Driving - A State-of-the-Art", Application of Stress Wave Theory to Piles, edited by $\mathrm{H}$. Bredenberg, Rotterdam: A.A. Balkema,1981. pp. 131-161.

[3] E.A. Smith, "Pile Driving Analysis by the Wave Equation", Transactions, ASCE, Vol. 127, 1962, pp. 1145-1193. 
[4] M.F. Randolph, and A.J. Deeks. "Dynamic and Static Soil Models for Axial Pile Response. Application of Stress wave Theory to Piles, edited by H. Bredenberg. Rotterdam: A.A. Balkema.1981, pp. 3-14.

[5] F.E. Richart, R.D. Woods, J.R. Hall, Vibrations of Soils and Foundations, Prentice-Hall, 1970.

[6] C.H.Yang, An exploratory study to estimate pile bearing capacity via seismic measurements, Dissertation submitted as partial fulfillment of the requirements of the Ph.D. degree in Civil Engineering, The Pennsylvania State University, 1997.

[7] C.H. Dowding, Construction Vibrations, Prentice Hall, 1996.

[8] N. Tang, "Chinese Experience in Stress Wave Theory Applied to Pile Foundations", Application of Stress Wave Theory to Piles, edited by H. Bredenberg, Rotterdam: A.A. Balkema,1981, pp. 297-304. 